\title{
EFICIÊNCIA DE ÍNDICES DE SELEÇÃO UTILIZANDO A METODOLOGIA REML/ BLUP NO MELHORAMENTO DA CANA-DE-AÇÚCAR
}

\section{EFFICIENCY OF SELECTION INDEXES USING THE REML/BLUP PROCEDURE IN SUGARCANE BREEDING}

\author{
Cássia Ângela PEDROZO' \\ Flávio Rodrigo Gandolfi BENITES ${ }^{2}$ \\ Márcio Henrique Pereira BARBOSA ${ }^{3}$ \\ Marcos Deon Vilela de RESENDE ${ }^{4}$ \\ Felipe Lopes DA SILVA ${ }^{5}$
}

\begin{abstract}
RESUMO
Neste estudo objetivou-se comparar a eficiência de três índices de seleção construídos a partir de componentes de variância estimados e valores genotípicos preditos pela metodologia REML/BLUP, em três populações $(A, B$ e $C)$ de primeira geração clonal de cana-de-açúcar. As populações foram avaliadas na área experimental do Centro de Pesquisa e Melhoramento da Cana-de-açúcar - CECA da Universidade Federal de Viçosa, utilizando o delineamento experimental em blocos aumentados. Os caracteres número de colmos por metro (NCM), massa média do colmo (MMC), teor de sólidos solúveis totais (BRIX) e produção de BRIX por hectare (TBH) foram avaliados nos ciclos de cana-planta e de cana-soca nas populações $A$ e $B$ e apenas no ciclo de cana-planta na população $C$. Os três primeiros caracteres foram utilizados para construir os seguintes índices de seleção: clássico, multiplicativo e com base na soma de postos. As análises genético-estatísticas foram realizadas pelos programas GENES e Selegen - REM/BLUP. O índice multiplicativo foi o que mostrou maior eficiência de seleção, uma vez que, apresentou maior coeficiente de coincidência de genótipos selecionados com a estratégia de seleção para o caráter TBH, maiores ganhos diretos de seleção e maiores ganhos indiretos para TBH. No entanto, a eficiência de seleção de determinado índice deve ser considerada como característica inerente à população estudada.
\end{abstract}

Palavras-chave: valores genotípicos preditos; geração clonal; modelos mistos.

\begin{abstract}
This study aimed to compare the efficiency of three selection indexes constructed from the estimated variance components and predicted genotypic values by the REML/BLUP procedure, on three first clonal populations in sugarcane. The populations were evaluated at the experimental area of the Sugarcane Research and Breeding Center - CECA of the Federal University of Viçosa, using the augmented block design. The traits number of stems per meter (NSM), average stem mass (ASW), total soluble solids content (BRIX) and production of BRIX per hectare (TBH) were evaluated in the cane plant and sugar cane ratoon-cycle in the $A$ and $B$ populations and the cane plant in the $C$ population. The first three traits were used to construct the following selection indexes: classic index, multiplicative index and the index based on sum of ranks. The genetic-statistics analyses were carried by the GENES and Selegen - REM/BLUP programs. The multiplicative index showed higher selection efficiency as it presented larger coefficient of coincidence of genotypes selected with the selection strategy for TBH, larger direct gains from selection and larger indirect gains for TBH. However, the rate of selection efficiency of a certain index should be considered as an intrinsic characteristic of the studied population.
\end{abstract}

Key-words: predicted genotypic values; clonal generation; mixed model.

\footnotetext{
Engenheira Agrônoma. Aluna de doutorado do Programa de Pós-graduação em Genética e Melhoramento. Departamento de Fitotecnia. Universidade Federal de Viçosa (UFV). Rua João Alfredo, n.44, ap.02. Bairro: Ramos. CEP: 36570-000. Viçosa, MG - Brasil. E-mail: cassiapedrozo@yahoo.com.br. Autora para correspondência.

${ }^{2}$ Engenheiro Agrônomo. Aluno de pós-doutorado do Programa de Pós-graduação em Genética e Melhoramento. Departamento de Fitotecnia. Universidade Federal de Viçosa, Viçosa, MG - Brasil. E-mail: frgbenites@yahoo.com.br;

${ }^{3}$ Engenheiro Agrônomo. Dr. Professor Titular. Departamento de Fitotecnia. Universidade Federal de Viçosa, Viçosa, MG - Brasil. E-mail: barbosa@ufv.br.

${ }^{4}$ Engenheiro Agrônomo. Pesquisador da Embrapa Florestas. Colombo, PR - Brasil. E-mail: marcos.deon@gmail.com.

${ }^{5}$ Engenheiro Agrônomo. Aluno de doutorado do Programa de Pós-graduação em Genética e Melhoramento. Departamento de Fitotecnia. Universidade Federal de Viçosa, Viçosa, MG - Brasil. E-mail: ipells@yahoo.com.br.
} 


\section{INTRODUÇÃO}

O principal objetivo no melhoramento da cana-de-açúcar é a obtenção de variedades que agreguem uma série de atributos favoráveis, tanto no âmbito agronômico, como no industrial. Uma das estratégias utilizadas nas fases iniciais de seleção no processo de melhoramento genético desta cultura se refere à seleção indireta (via caracteres correlacionados) para produção de açúcar ou teor de sólidos solúveis totais (BRIX), a qual facilita a obtenção de ganhos para estes caracteres, em relação ao uso da seleção direta. No entanto, sabese que a seleção baseada em um ou em poucos caracteres pode se mostrar inadequada, uma vez que, correlações negativas entre os caracteres de interesse podem ser observadas. Uma alternativa, neste caso, seria a adoção da estratégia de índice de seleção, que foi inicialmente proposta por Smith (1936) e Hazel (1943), e tem como finalidade a seleção de diversos caracteres simultaneamente.

$\mathrm{Na}$ literatura são encontrados vários índices de seleção, os quais podem ser utilizados no melhoramento de plantas (Cruz et al., 2004). Estes índices são obtidos como combinações lineares das medidas de diversos caracteres, podendo ser eficientes, uma vez que, permitem a avaliação de todas as informações disponíveis, atribuem diferentes pesos aos caracteres estudados e valorizam atributos julgados de maior importância pelo pesquisador (Falconer, 1987).

Apesar da aplicabilidade dos índices de seleção ter sido demonstrada para diversas culturas (Barbosa \& Pinto, 1998; Granate et al., 2002; Martins et al., 2003), em cana-de-açúcar poucos estudos empregando esta estratégia são encontrados na literatura (Singh \& Khan, 1998; Pillai \& Ethirajan, 1993). Tais índices são geralmente construídos a partir de estimativas de parâmetros genéticos e médias fenotípicas obtidas pelo método da análise de variância. No entanto, uma alternativa a ser empregada na construção de índices e que pode resultar em um processo de seleção mais acurado é o emprego de componentes de variância estimados por máxima verossimilhança restrita (REML) e valores genéticos ou genotípicos preditos pelo melhor preditor linear não viciado (BLUP) (Resende, 2002b).

Neste estudo objetivou-se comparar a eficiência de três índices de seleção construídos a partir de componentes de variância estimados e valores genotípicos preditos pela metodologia REML/BLUP, em três populações de cana-de-açúcar.

\section{MATERIAL E MÉTODOS}

O material experimental utilizado neste estudo consistiu de três populações de primeira geração clonal, correspondente à segunda fase de seleção (Fase T2) do programa de melhoramento da cana-de-açúcar da Rede Interuniversitária para Desenvolvimento do Setor Sucroalcooleiro (RIDESA). Cada população foi constituída por um conjunto de genótipos de primeira geração clonal selecionados de uma série de cruzamentos, sendo cada série realizada em anos distintos. Essas informações podem ser observadas na Tabela 1. Os cruzamentos foram realizados na estação de Floração e Cruzamento da Serra do Ouro, localizada no município de Murici, Estado de Alagoas, e situada à latitude $9^{\circ} 13^{\prime} \mathrm{S}$, longitude $35^{\circ} 50^{\prime} \mathrm{W}$ e à altitude de 450 a $500 \mathrm{~m}$.

Os experimentos descritos na Tabela 1 foram instalados no Centro de Pesquisa e Melhoramento da Cana-de-açúcar - CECA da Universidade Federal de Viçosa, o qual se localiza no município de Oratórios - MG, à latitude $20^{\circ} 25^{\prime} \mathrm{S}$, longitude $42^{\circ} 48^{\prime}$ ' W e a 494 m de altitude.

$O$ delineamento experimental utilizado foi o de blocos aumentados, sendo as populações A, B e C constituídas por 48, 6 e 24 blocos, respectivamente. Como genótipos comuns foram utilizadas as variedades RB72454 e RB835486. Cada genótipo foi plantado em um sulco de 5,0 m de comprimento, espaçados entre si de $1,40 \mathrm{~m}$. A adubação e demais tratos culturais foram realizados de acordo com a recomendação para a cultura da cana-de-açúcar na região.

Os caracteres número de colmos por metro$\mathrm{NCM}$, massa média do colmo-MMC $(\mathrm{kg})$, teor de sólidos solúveis totais-BRIX e produção de BRIX por hectare - TBH $\left(\mathrm{t} \mathrm{ha}^{-1}\right)$ foram avaliados nos ciclos de cana-planta e cana-soca para as populações A e B e apenas no ciclo de cana-planta para a população C. Para os caracteres MMC e BRIX foram avaliados, aleatoriamente, três colmos industrializáveis por parcela.

Foram utilizados os caracteres NCM, MMC e BRIX para construção dos seguintes índices de seleção: clássico (Smith, 1936; Hazel, 1943), multiplicativo (Subandi et al., 1973) e com base na soma de postos (Mulamba \& Mock, 1978). No índice clássico o desvio-padrão genético foi estabelecido como peso econômico. A intensidade de seleção praticada foi de $7,75 \%$, a qual foi escolhida de acordo com aquela praticada (aproximadamente $10 \%$ ) para a primeira geração clonal nos programas de melhoramento da cana-de-açúcar.

Com o objetivo de garantir a confiabilidade das variâncias estimadas e, portanto, viabilizar o estudo dos índices de seleção, anteriormente à obtenção destes, foi realizado o diagnóstico de multicolinearidade dos caracteres, por meio do programa computacional GENES (Cruz, 2001).

Todos os procedimentos necessários, para a construção dos índices de seleção, foram realizados pelo programa Selegen - REM/BLUP (Resende, 2002a). Os modelos genéticoestatísticos utilizados para estimação dos componentes de variância e predição dos valores genotípicos são descritos a seguir:

Modelo genético-estatístico utilizado para avaliação das populações $A$ e $B$, separadamente:

$y=X m+Z g+W b+T i+Q p+e$

Em que: y: vetor de dados; $m$ : vetor dos efeitos fixos das medições somados a media geral; $\mathrm{g}$ : vetor dos efeitos aleatórios de genótipos; b: vetor dos efeitos aleatórios de blocos; i: vetor dos efeitos 
aleatórios da interação genótipos $x$ medições; $p$ : vetor dos efeitos de ambiente permanente (parcelas no caso) (aleatórios); X, Z, W, T e Q: matrizes de incidência que associam os efeitos do modelo aos dados; e: vetor de erros ou resíduos (aleatórios). Modelo genético-estatístico utilizado para avaliação da população $C$ :

$$
y=X f+Z g+W b+e
$$

Em que: $y$ : vetor de dados; $f$ : vetor do efeito fixo das medições somados à media geral; g: vetor dos efeitos aleatórios de genótipos; $b$ : vetor dos efeitos aleatórios de blocos; X, Z, W: matrizes de incidência que associam os efeitos do modelo aos dados; e: vetor de erros ou resíduos (aleatórios).

Os índices de seleção clássico (IC), multiplicativo (IM) e com base na soma de postos (ISR), construídos a partir da metodologia REML/ BLUP são sucintamente apresentados a seguir: $I C=((p \times N C M) \times(V G \times N C M))+((p \times M M C) \times$ $(V G \times M M C))+((p \times B R I X) \times(V G \times B R I X))$

$\mathrm{IM}=(\mathrm{VG} \times \mathrm{NCM}) \times(\mathrm{VG} \times \mathrm{MMC}) \times(\mathrm{VG} \times \mathrm{BRIX})$ $x$ BRIX $I S R=(r \times V G \times N C M)+(r \times V G \times M M C)+(r \times V G$

Em que: p: peso econômico estabelecido para o caráter; VG: valor genotípico predito; r: posto do genótipo.

A eficiência dos índices de seleção foi obtida por meio do coeficiente de coincidência dos genótipos selecionados por estes índices e dos genótipos selecionados com base no caráter TBH. Adicionalmente, foram estimados os ganhos genéticos obtidos pelos índices de seleção, o ganho com a seleção direta para o caráter TBH e os ganhos indiretos para este caráter via seleção pelos índices. Os ganhos genéticos estimados foram obtidos pela média dos VG dos indivíduos selecionados.

\section{RESULTADOS E DISCUSSÂO}

Foi detectada presença de multicolinearidade fraca (dados não apresentados) entre os caracteres, para todas as populações avaliadas neste estudo, não constituindo, portanto, em um problema para a obtenção dos índices de seleção.

As estimativas de herdabilidade individual no sentido amplo $\left(\mathrm{h}^{2}{ }_{\mathrm{g}}\right)$, dos desvios-padrão (s) associados a cada estimativa de $\mathrm{h}^{2}$ e das médias gerais, considerando todos os caracteres avaliados nas três populações $\left(P_{A}, P_{B}\right.$ e $\left.P_{C}\right)$ encontram-se na Tabela 2. Exceto para o NCM na $P_{C}(0,6814)$, todas as demais estimativas de $\mathrm{h}^{2}$ foram baixas. Baixas estimativas de $h^{2}$ na primeira geração clonal, onde se avaliaram os ciclos de cana-planta e cana-soca, foram também encontradas em um estudo realizado por Pedrozo (2006).

Dentre as demais populações, a $P_{c}$ foi a que apresentou as maiores estimativas de $\mathrm{h}^{2}{ }_{\mathrm{c}}$. Esta superioridade de estimativas observadas para a PC pode ter ocorrido em conseqüência de esta população ter sido avaliada apenas no ciclo de canaplanta. Neste caso, a variância genótipica contempla também, a variância devido ao ambiente permanente, tornando os valores de herdabilidade superestimados. Além disso, segundo Falconer \& Mackay (1997), a herdabilidade é uma propriedade não somente de um caráter, mas também, da população, das circunstâncias ambientais as quais os indivíduos são submetidos e da forma em que o fenótipo é mensurado.

TABELA 1 - Número de genótipos (NG), ano de realização dos cruzamentos (ARC) e ano de instalação dos experimentos (AIE) referentes a três populações (A, B e C) avaliadas na primeira geração clonal em cana-de-açúcar.

\begin{tabular}{clll}
\hline População & NG & ARC & AIE \\
\hline A & 944 & 1999 & 2001 \\
B & 383 & 2000 & 2002 \\
C & 548 & 2002 & 2004 \\
\hline
\end{tabular}

Medições realizadas em diferentes ciclos, como no caso das $\mathrm{P}_{\mathrm{A}}$ e $\mathrm{P}_{\mathrm{B}}$, tendem a elevar a acurácia das estimativas de parâmetros genéticos, tais como a herdabilidade. Ferreira et al. (2005), ao avaliar três caracteres em genótipos de cana-de-açúcar concluíram que, três medições possibilitariam a discriminação dos genótipos com $80 \%$ de probabilidade de se estar fundamentado no valor real de cada um deles.
Os caracteres que apresentaram as maiores estimativas de $\mathrm{h}^{2}$ foram o $\operatorname{NCM}(0,2758$, 0,2906 e 0,6814 para $P_{A}, P_{B}^{g}$ e $P_{C}$, respectivamente) $\mathrm{e}$ o $\operatorname{BRIX}\left(0,2715,0,2379\right.$ e 0,3750 para $P_{A}, P_{B}$ e $P_{C}$, respectivamente). Em uma revisão realizada por Skinner et al. (1987), concluíram que o BRIX é um dos caracteres de maior herdabilidade nas fases iniciais de seleção no melhoramento da cana-deaçúcar. 
PEDROZO, C.A. et al. Eficiência de índices de seleção...

TABELA 2 - Estimativas de herdabilidade individual no sentido amplo $\left(\mathrm{h}^{2}{ }_{\mathrm{g}}\right)$, dos desvios-padrão (s) associados a cada estimativa de $h^{2}$ e da média geral $(\mathrm{m})$ para massa média de colmos (MMC), número de colmos por metro (NCM), teor de sólidos solúveis totais (BRIX) e produção de BRIX por hectare $(T B H)$, avaliados em três populações $\left(P_{A}, P_{B}\right.$ e $\left.P_{C}\right)$ de primeira geração clonal em cana-de-açúcar.

\begin{tabular}{|c|c|c|c|c|c|}
\hline \multirow{2}{*}{ População } & \multirow{2}{*}{ Parâmetros } & \multicolumn{4}{|c|}{ Caracteres } \\
\hline & & MMC (kg) & $\mathrm{NCM}$ & BRIX & TBH $\left(\mathrm{t} \mathrm{ha}^{-1}\right)$ \\
\hline & $\mathrm{h}_{\mathrm{g}}^{2}$ & 0,1579 & 0,2758 & 0,2715 & 0,1554 \\
\hline \multirow[t]{3}{*}{$A$} & s & 0,0248 & 0,0327 & 0,0325 & 0,0246 \\
\hline & $\mathrm{m}$ & 1,14 & 10,14 & 19,43 & 9,37 \\
\hline & $h_{g}^{2}$ & 0,2087 & 0,2906 & 0,2379 & 0,1126 \\
\hline \multirow[t]{3}{*}{ B } & $\mathrm{s}$ & 0,0432 & 0,0509 & 0,0462 & 0,0318 \\
\hline & $\mathrm{m}$ & 1,35 & 9,65 & 19,34 & 14,37 \\
\hline & $h_{g}^{2}$ & 0,2616 & 0,6814 & 0,3750 & 0,2117 \\
\hline \multirow[t]{2}{*}{ C } & s & 0,0592 & 0,0955 & 0,0708 & 0,0532 \\
\hline & $\mathrm{m}$ & 1,48 & 6,82 & 19,18 & 22,37 \\
\hline
\end{tabular}

Os desvios-padrão (s) associados a cada estimativa de $\mathrm{h}_{\mathrm{g}}$ (Tabela 2 ) variaram de moderados a baixos, revelando que todas as herdabilidades estimadas são estatisticamente maiores que zero. Bressiani (1993) encontrou baixos desvios associados às estimativas de herdabilidade no sentido restrito obtidas para a primeira geração clonal.

$\mathrm{Na}$ Tabela 3 encontram-se os coeficientes de coincidência de genótipos selecionados por meio dos índices de seleção clássico (IC), multiplicativo (IM) e com base na soma de postos (ISR) e de genótipos selecionados com base no caráter $\mathrm{TBH}$. Ao se considerar apenas as coincidências entre os índices de seleção, estes coeficientes foram em geral baixos. Os maiores coeficientes foram encontrados entre os índices IM e IC nas $\mathrm{P}_{\mathrm{A}}$ e $\mathrm{P}_{\mathrm{C}}$ $(0,63$ e 0,57 , respectivamente) e entre os índices IC e ISR e entre IM e ISR, ambos na $\mathrm{P}_{\mathrm{B}}(0,47)$. Quanto maior o coeficiente de coincidência entre dois índices de seleção, maior será a concordância dos resultados de seleção entre eles. Pelos resultados mostrados, pode-se observar que a concordância de seleção entre os índices variou de acordo com a população, fato que pode ter sido ocasionado pelas diferenças na constituição genética das populações avaliadas, uma vez que cada população foi constituída por um grupo distinto de genótipos.

Ao se comparar os índices de seleção e o caráter TBH, os maiores coeficientes de coincidência de genótipos selecionados foram obtidos para o IM em todas as populações estudadas $\left(0,82,0,70\right.$ e 0,47 para $P_{A}, P_{B}$ e $P_{C}$, respectivamente) (Tabela 3 ). A maior coincidência de genótipos selecionados entre o IM e o caráter TBH era esperado, uma vez que existe uma determinada relação entre estas duas estratégias de seleção. O índice multiplicativo é construído pela multiplicação dos valores padronizados de cada caráter avaliado (Subandi et al., 1973) e o TBH é resultante da associação dos caracteres altura, diâmetro, densidade e número de colmos e BRIX (Bressiani et al., 2001). Os três primeiros caracteres são determinantes da massa média de colmos (Chang \& Milligan, 1992; Ferreira et al., 2007).

$\mathrm{Na}$ Tabela 4 são apresentados os ganhos genéticos totais esperados com a seleção ao se utilizar os índices e a seleção direta para TBH, estes sendo conferidos pela estimativa denominada GS, e a seleção indireta para TBH via índices de seleção (GSI). Quanto aos ganhos obtidos pela seleção direta em $\mathrm{TBH}$, a $\mathrm{P}_{\mathrm{A}}$ foi a que apresentou maior ganho $(20,68 \%)$.

Em todas as populações o IM foi o índice que proporcionou maior ganho $(24,77,22,15 \mathrm{e}$ $48,02 \%$ para $P_{A}, P_{B}$ e $P_{C}$, respectivamente). No entanto, de acordo com Cruz et al. (2004) diferentes índices representam diferentes alternativas de seleção e, consequentemente, de ganhos.

Falconer (1987) relata que algumas vezes é possível alcançar progressos mais rápidos, por meio de resposta correlacionada, do que pela seleção do caráter diretamente desejado. Em relação aos ganhos indiretos esperados para $\mathrm{TBH}$ via seleção pelos índices, maiores ganhos foram obtidos via IM para as $\mathrm{P}_{A}$ e $\mathrm{P}_{B}(20,17$ e $12,90 \%$, respectivamente), e via ISR para a $P_{C}(10,70 \%)$. No entanto, quanto à $P_{C}$, vale ressaltar que as estimativas de ganho obtidas podem estar viesadas, uma vez que, para esta população, como discutido anteriormente, as estimativas de herdabilidade podem estar superestimadas. 
PEDROZO, C.A. et al. Eficiência de índices de seleção...

TABELA 3 - Coeficientes de coincidência dos genótipos selecionados considerando os índices de seleção Clássico (IC), Multiplicativo (IM) e baseado na Soma de Postos (ISR) e o caráter produtividade de $\operatorname{BRIX}(T B H)$ para três populações $\left(P_{A}, P_{B}\right.$ e $\left.P_{C}\right)$ de primeira geração clonal em cana-de-açúcar.

\begin{tabular}{clccc}
\hline \multirow{2}{*}{ População } & & IM & ISR & TBH \\
\hline \multirow{2}{*}{ A } & IC & 0,63 & 0,23 & 0,47 \\
& IM & - & 0,47 & 0,82 \\
& ISR & & - & 0,53 \\
\hline B & IC & 0.40 & 0,47 & 0,33 \\
& IM & - & 0,47 & 0,70 \\
& ISR & & - & 0,50 \\
\hline & IC & 0,57 & 0,20 & 0,07 \\
& IM & - & 0,37 & 0,47 \\
& ISR & & - & 0,37 \\
\hline
\end{tabular}

TABELA 4 - Ganhos genéticos (GS) esperados pela seleção utilizando os índices clássico (IC), multiplicativo (IM) e baseado na soma de Postos (ISR), seleção direta para a produtividade de BRIX (TBH) e ganhos genéticos esperados pela seleção indireta (GSI) para TBH via seleção pelos índices, em três populações $\left(\mathrm{P}_{\mathrm{A}}, \mathrm{P}_{\mathrm{B}}\right.$ e $\left.\mathrm{P}_{\mathrm{C}}\right)$ de primeira geração clonal em cana-de-açúcar.

Estratégias de seleção

\begin{tabular}{cccccc} 
& & & & & \\
\hline & & & & & \\
\hline & $P_{\mathrm{B}}$ & $\mathrm{P}_{\mathrm{C}}$ & $\mathrm{P}_{\mathrm{A}}$ & $\mathrm{P}_{\mathrm{B}}$ & $\mathrm{PC}_{\mathrm{C}}$ \\
\hline 22,90 & 4,85 & 9,59 & 16,30 & 10,32 & 4,95 \\
24,77 & 22,15 & 48,02 & 20,17 & 12,90 & 7,57 \\
15,17 & 9,38 & 20,11 & 18,37 & 11,91 & 10,7 \\
20,68 & 13,32 & 12,47 & - & - & -
\end{tabular}

Os resultados dos ganhos indiretos para TBH observados neste estudo mostram que, o IM, de uma forma geral, foi o índice de maior eficiência para seleção daquele caráter. Nas populações A, B e C os ganhos indiretos via IM conduziram a, respectivamente, $98 \%, 97 \%$ e $61 \%$ do ganho genético com a seleção direta para TBH.

A seleção indireta pode ser mais vantajosa que a direta quando há dificuldades técnicas ao se aplicar seleção direta sobre o caráter desejado (Falconer, 1987). Segundo Bressiani et al. (2001) o TBH é determinado pela produção de colmos $(\mathrm{TCH})$ e pelo BRIX. Geralmente, em termos práticos, o BRIX é determinado de colmos amostrados aleatoriamente, dentro de cada parcela, enquanto que, o TCH é obtido por um procedimento bastante trabalhoso, onde cada parcela precisa ser inteiramente colhida e, posteriormente, pesada. Pelos resultados encontrados neste estudo, o índice de seleção multiplicativo, com base no NCM, na MMC e no BRIX poderia ser utilizado como alternativa à seleção direta para $\mathrm{TBH}$, facilitando, desta forma, a seleção de genótipos para este caráter.
Pelos resultados apresentados anteriormente, pode se observar que o índice multiplicativo foi o que mostrou maior eficiência de seleção, uma vez que, apresentou maior coeficiente de coincidência de genótipos selecionados com a estratégia de seleção para o caráter $\mathrm{TBH}$, maiores ganhos diretos de seleção e maiores ganhos indiretos para TBH. No entanto, a eficiência de seleção ao se utilizar um determinado índice, assim como as estimativas de herdabilidade, são características inerentes à população estudada.

\section{CONCLUSÕES}

O índice multiplicativo foi o que mostrou maior eficiência de seleção de genótipos superiores de cana-de-açúcar, podendo aumentar a chance de sucesso em programas de melhoramento desta cultura.

\section{AGRADECIMENTO}

À FAPEMIG pela concessão da bolsa de pós-doutorado. 


\section{REFERÊNCIAS}

1. BARBOSA, M. H. P.; PINTO, C. A. B. P. Eficiência de índices de seleção na identificação de clones superiores de batata. Pesquisa Agropecuária Brasileira, v. 33, n. 2, p. 149-156, 1998.

2. BRESSIANI, J. A. Herdabilidade e repetibilidade na cultura da cana-de-açúcar. 1993. 66 f. Dissertação (Mestrado em Genética e Melhoramento de Plantas) - Escola Superior de Agricultura Luiz de Queiroz, Piracicaba, 1993.

3. BRESSIANI, J. A. et al. Combining ability in eight selected clones of sugarcane (Saccharum sp.). Crop Breeding and Applied Biotechnology, v. 2, n. 3, p. 411-416, 2001.

4. CHANG, Y. S., MILLIGAN, S. B. Estimating the potential of sugarcane families to produce elite genotypes using univariate cross prediction methods. Theoretical and Applied Genetics, v. 84, p. 662-671, 1992.

5. CRUZ, C. D. Programa Genes: versão Windows; aplicativo computacional em genética e estatística. Viçosa: Editora UFV, 2001. 648 p.

6. CRUZ, C. D.; REGAZZI, A. J.; CARNEIRO, P. C. S. Modelos biométricos aplicados as melhoramento genético. 3. ed. Viçosa: UFV, 2004. v. 1. 480 p.

7. FALCONER, D. S. Introdução à genética quantitativa. Tradução de M.A. SILVA; J.C. SILVA. Viçosa: UFV, 1987. 279p.

8. FALCONER, D. S.; MACKAY, T. F. C. Introduction to quantitative genetics. Edinburgh: Longman: 1997. 464 p.

9. FERREIRA, A. et al. Repetibilidade e número de colheitas para seleção de clones de cana-de-açúcar. Pesquisa Agropecuária Brasileira, v. 40, n. 8, p. 761-767, 2005.

10. FERREIRA, F. M. et al. Relações fenotípicas e genotípicas entre componentes de produção em cana-de-açúcar. Bragantia, v. 66, n. 4, p. 605-610, 2007.

11. GRANATE, M. J.; CRUZ, C. D.; PACHECO, C. A. P. Predição de ganho genético com diferentes índices de seleção no milho pipoca CMS-43. Pesquisa Agropecuária Brasileira, v. 37, n. 7, p. 1001-1008, 2002.

12. HAZEL, L. N. The genetic basis for constructing selection indexes. Genetics, v. 28, p. 476-490, 1943.

13. MARTINS, I. S. et al. E. Eficiência da seleção univariada direta e indireta e de índices de seleção em Eucalyptus grandis. Revista Árvore, v. 27, n. 3, p. 327-333, 2003.

14. MULAMBA, N. N.; MOCK, J. J. Improvement of yield potential of the Eto Blanco maize (Zea mays L.) population by breeding for plant traits. Egyptian Journal of Genetics and Cytology, v. 7, p. 40-51, 1978.

15. PEDROZO, C. A. Eficiência da seleção em fases iniciais no melhoramento da cana-de-açúcar. $2006.111 \mathrm{f}$. Dissertação (Mestrado em Genética e Melhoramento) - Universidade Federal de Viçosa, Viçosa, 2006.

16. PILLAI, S. V.; ETHIRAJAN, A. S. Selection indices for sugarcane improvement at three stages of selection. Euphytica, v. 71, p. 155-159, 1993.

17. RESENDE, M. D. V. Software Selegen-REML/BLUP. Curitiba: Embrapa, 2002a. 67 p. (Documentos, 77).

18. RESENDE, M. D. V. Genética biométrica e estatística no melhoramento de plantas perenes. Brasília: Embrapa Informação Tecnológica, 2002b. 975 p.

19. SINGH, S. P.; KHAN, A. Q. Selection indices for cane yield in sugarcane. Indian Journal Genetics, v. 58, n. 3, p. 353$357,1998$.

20. SKINNER, J. C.; HOGARTH, D. M.; WU, K. K. Selection methods, criteria, and indices. In: HEINZ, D. J. (Ed.). Sugarcane improvement through breeding. Amsterdam: Elsevier, 1987. p. 409-453.

21. SMITH, H. F. A discriminant function for plant selection. Annual Eugenetic, v. 7, p. 240-250, 1936.

22. SUBANDI, W.; COMPTON, A.; EMPIG, L. T. Comparison of the efficiencies of selection indices for three traits in two variety crosses of corn. Crop Science, v. 13, p. 184-186, 1973.

Recebido em 30/04/2008 Aceito em 02/07/2008 